\title{
PRELIMINARY LIDAR EXPERIMENT TO STUDY THE BACKSCATTER AMPLIFICATION
}

\author{
Igor A. Razenkov", Victor A. Banakh \\ V.E. Zuev Institute of Atmospheric Optics, Tomsk, 634055, RUSSIA, *Email: lidaroff@iao.ru
}

\begin{abstract}
Long-term continuous measurements for detection relative backscatter amplification on a horizontal path of $2 \mathrm{~km}$ long are performed by using a specific micro pulse lidar. The laser beam path is limited by a solid obstacle. The lidar is located next to an ultrasonic anemometer that measures $3 \mathrm{D}$ wind velocity and temperature; the laser spot on the obstacle is observed by using a telephoto lens. The results showed that the backscatter amplification has a clear diurnal variation. Moreover, the backscatter amplification was completely absent in the morning and evening under neutral stratification in the atmospheric surface layer. At night and in the daytime there was a significant increase of the backscatter amplification coefficient.
\end{abstract}

\section{INTRODUCTION}

The backscatter amplification effect occurs in the turbulent atmosphere, when the probe laser beam and scattered light propagate along the same trajectory. The increase of the scattered light intensity fluctuations at the point of reception is the result of the correlation of direct and backward light waves. The point of reception must be located on the axis of the probe beam [1-3].

To register the relative backscatter amplification (RBSA) was build the lidar with beam expansion via receiving telescope [4]. RBSA-lidar has one transmitting channel and two receiving channels: on-axis receiving channel registers echo exactly on the axis of the laser beam, the off-axis channel registers the signal $200 \mathrm{~mm}$ apart from the probing beam axis. The system continuously works in Tomsk since the end of August 2014.

\section{EXPERIMENT}

Figure 1 shows an arrangement of the experiment for the lidar test. Lidar operates at the elevation angle close to horizontal. The laser beam is directed to the wall at the distance of about $2 \mathrm{~km}$. A modified web camera mounted on the Maksutov telescope with a focal length of one meter is located next to the lidar. The mast with the ultrasonic anemometer $[5,6]$ is located above the RBSA-lidar on the roof of the building.

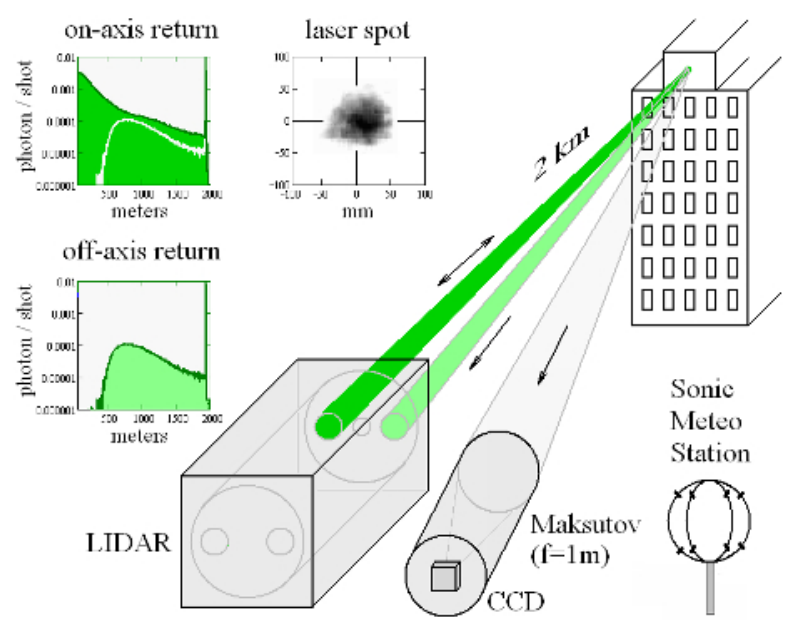

Fig. 1. Experimental setup.

Figure 1 also shows on-axis return, off-axis return, and laser spot on the wall. Experiments are done in the city Tomsk. Figure 2 shows a Google map. The lidar is located in the building of the Institute of Atmospheric Optics. Sounding path (straight yellow line) passed over the city (roads, small river, buildings, and trees).

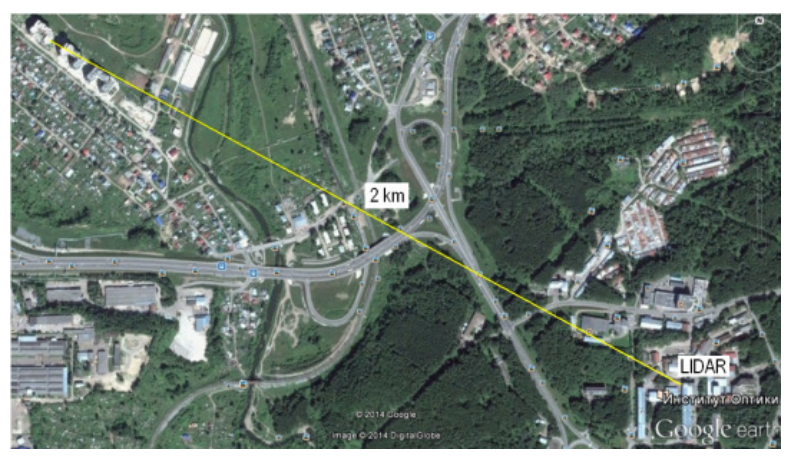

Fig. 2. A map of the experiment site (Tomsk, Russia). 
The lidar operates in the photon counting mode, the counts accumulation time interval is ten minutes. Data processing for the instrument is described in [4]. Fiber laser pulse repetition rate is $50 \mathrm{KHz}$. Each measurement cycle contains 30 million shots. Just an average was taken only a couple signal photons per shot. To improve statistics the data are averaged over a $50 \mathrm{~m}$ range intervals. The statistical error for the returns from the farthest range bin is less than $1 \%$. Note that off-axis return is equal to zero up to ranges of approximately $500 \mathrm{~m}$ due to lidar's geometry. The data acquired from ranges from $0.9 \mathrm{~km}$ to $2 \mathrm{~km}$ are considered to be reliable.

The coefficient $N$ of relative backscatter amplification is determined from the ratio of onaxis and off-axis returns. The instrument allows measure the coefficient $N$ with an accuracy of $\pm 5 \%$, even when the changes of the backscattering coefficient along the sounding path are $>100 \%$.

\section{DATA EXAMPLES}

The RBSA-lidar continuously operated during a half-year since August 2014. Figure 3 shows example of a two-day recording of the amplification coefficient $N(2 \mathrm{~km})$ at the distance up to $2 \mathrm{~km}$ in clear atmosphere in September 2014.
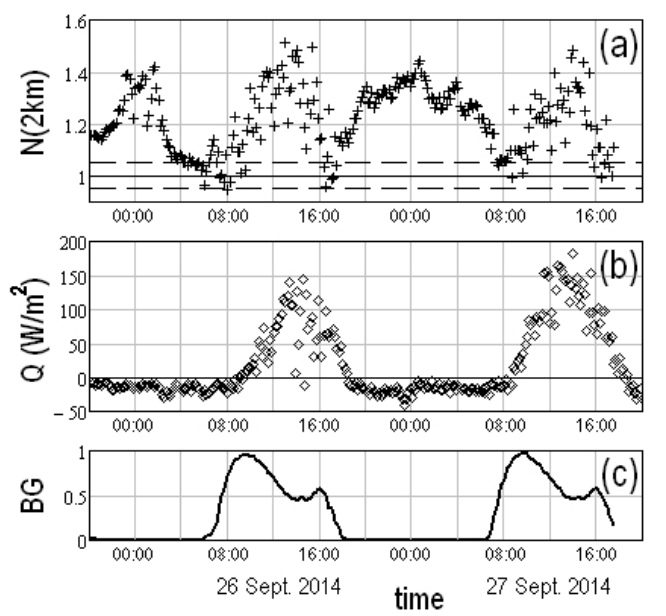

Fig. 3. The typical two days time series of (a) relative backscatter amplification $N$ in clear conditions. (b) turbulent heat flux $Q$, and (c) the magnitude of the normalized background $B G$. Data are recorded on 26 and 27 September 2014. Hereinafter, time is local.

The diurnal variation of the $N$ is clearly seen. Coefficient $N$ is equal to unit twice a day within a
$10 \%$ error of the accepted value. This happens in the mornings at $8 \mathrm{am}$ and in the evenings at $5 \mathrm{pm}$ of local time. It follows from Fig. 3(b) that at about the same time the turbulent heat flux $Q$ crossed the zero line. The third graph (c) shows the normalized values of the background $B G$, which was defined as an off-set of the lidar returns. The appearance of a second "evening" of the maximum in the curve $B G$ is due to the fact that the lidar path was directed to the North-West.

At day time and night time turbulent heat fluxes have opposite signs and in absolute value differ from each by the factor of about six. In this case, the amplification coefficient at the day and night increased to approximately the same value of 1.4. Also Fig. 3 shows that the data spread in the daytime is larger than that at night time.

Figure 4 shows another example of data obtained in 4 days period from 1 to 4 October 2014. Clear day is only on October 2 . We can see two clear maxima in the behavior of the $N$ which is similar to the data in Fig. 3. Some clouds appear on October 3, and the coefficient $N$ and turbulent flux $Q$ are smaller. It was cloudy on October 1 and 4; the heat flux $Q$ and the amplification of $N$ are even smaller. Note that we should not expect a very high correlation between RBSA-lidar data with the data of the anemometer, because it was located at the lidar site, but away from measuring volume.

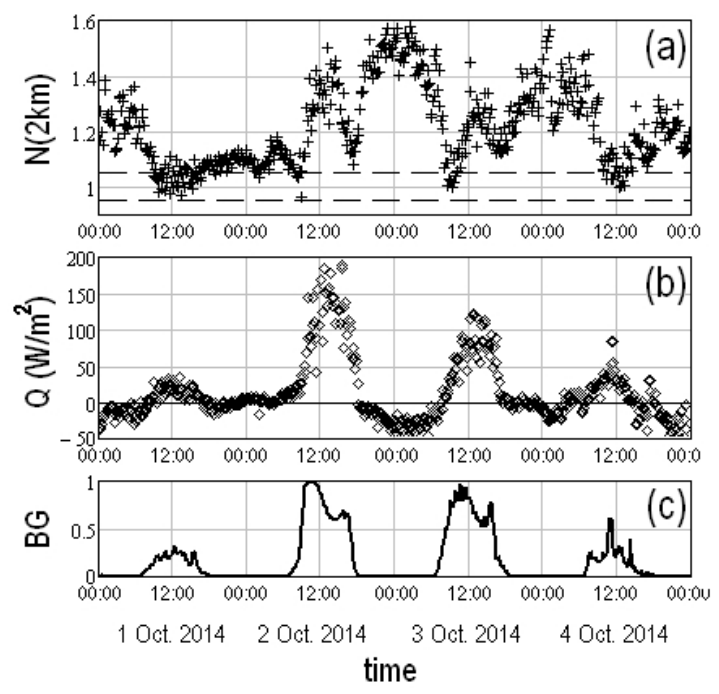

Fig. 4. Four days time series of (a) backscatter amplification $N$, (b) turbulent heat flux $Q$, and (c) the magnitude of the background $B G$ when changing cloud amount. Data are recorded starting on October 12014. 
Figure 5 shows the correlation between the coefficient $N$ and the turbulent heat flux $Q$ for the data in Fig. 4. A sharp increase of the amplification factor $N$ is seen under the stable temperature stratification for $Q<0$ (night time). With the increase of the heat flux for $Q>0$ (day time) there is a slow increase of $N$.

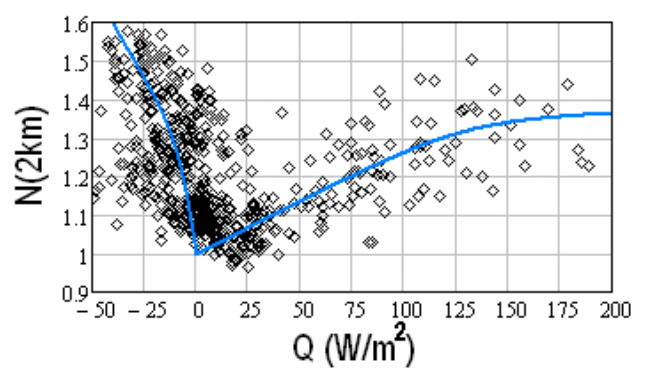

Fig. 5. Correlation plot of the amplification coefficient $N$ vs. the turbulent heat flux $Q$. Interpolation is shown by the solid blue line. Was used data from the Fig. 4 . Data were obtained from 1 to 4 October 2014.

For explanation of this we can assume that in a day time the radius of coherence of the laser beam is smaller than the Fresnel zone and less than the size of the aperture of the receiver respectively $[3,7]$. As a result, the averaging effect of the receiving aperture is stronger under larger turbulence intensity; this reduces the resulting estimate of the coefficient $N$. In the RBSA-lidar data analysis, it may make sense to consider separately "night" and "day" turbulence.

Figure 6 presents the data obtained in winter time. The atmosphere was not very clean on these days, but according to our lidar data, the visibility was more than $6 \mathrm{~km}$ in the presence of some clouds. Fig. 6 shows (a) the amplification factor $N$, (b) standard deviation of the centre of gravity of the laser spot (RMS CG) at a distance of $2 \mathrm{~km}$, (b) the structural constant of refractive index $C_{n}{ }^{2}$ and (d) the magnitude of the background $B G . R M S C G$ data were measurable only in the dark time by recording the jitter of the image of the laser spot by the camera sensor (see Fig. 1). $C_{n}{ }^{2}$ was calculated from anemometer's data [6].

Figure 7 shows (a) the correlation between the factor $N$ and the beam jitter $R M S C G$, and (b) between factor $N$ and the structure constant of refractive index $C_{n}^{2}$ for the data in Fig. 6.

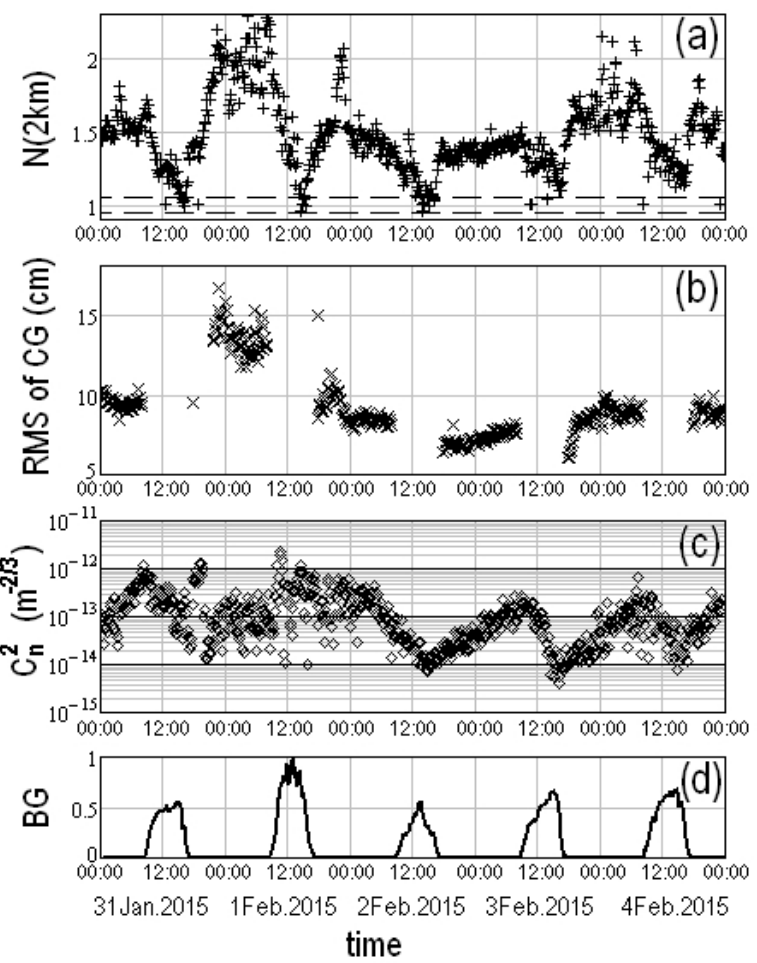

Fig. 6. Five days time series of (a) relative backscatter amplification $N(2 \mathrm{~km})$, (b) mean square displacement of the centre of gravity of the laser spot $R M S C G$ on the wall at a distance of $2 \mathrm{~km}$, (c) the structure constant of the refractive index $C_{n}{ }^{2}$ and (d) the magnitude of the normalized background $B G$. Data were obtained from 31 January to 4 February 2015.
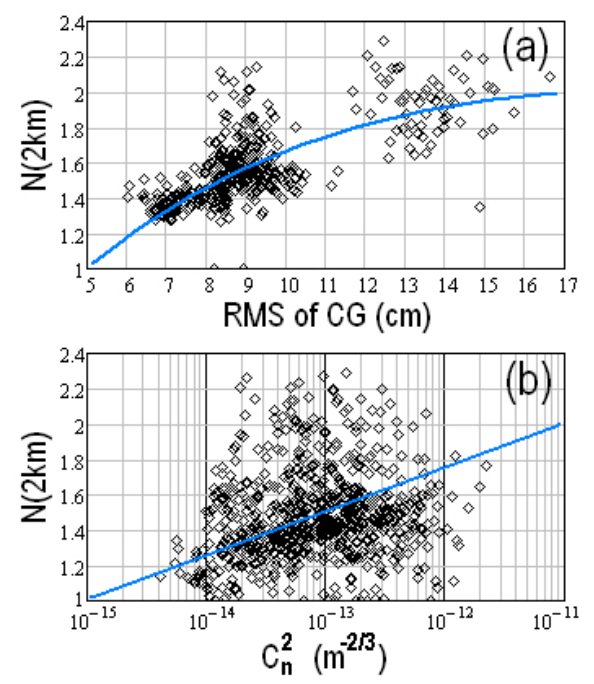

Fig. 7. Correlation plots of (a) factor $N$ vs. laser spot $R M S C G$, and (b) factor $N$ vs. the structure constant of refractive index $C_{n}{ }^{2}$. Fitting curves is shown with the solid blue line. Was used data from the Fig. 6. Data were obtained from 31 January to 4 February 2015. 
A positive correlation for both graphs in Fig. 7 should be noted. Fig. 7(b) shows a large spread of points due to all day time and night time data were putted on the one correlation plot. Was mentioned above that day time and night time behavior of the RBSA measurements is different.

Figure 8 presents laser spots at $2 \mathrm{~km}$ and shows beam's intensity, shape, and position changes. When turbulence is higher (a) the motion of laser spot is larger than when turbulence in lower $(b, c)$.

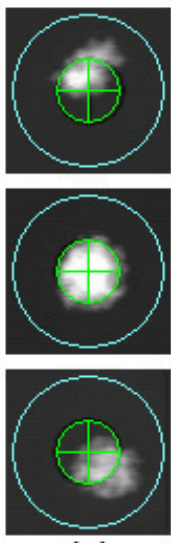

(a)

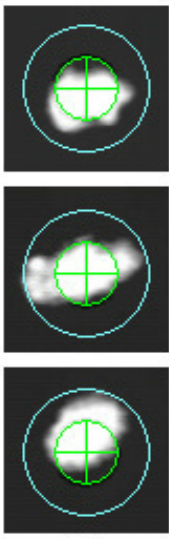

(b)

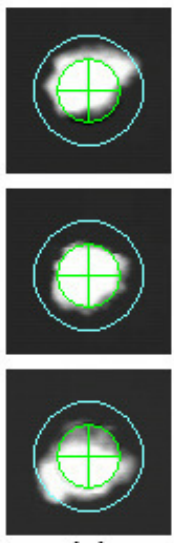

(c)
Fig. 8. The beam spot at $2 \mathrm{~km}$. Sets of three random images from data were obtained on the night (a) 1 February 2015, (b) 2 February 2015, and (c) 3 February 2015. Big blue circles are RMS CG from Fig. 6(b) plus beam size $100 \mathrm{~mm}$ (small green circle with cross).

\section{CONCLUSION}

The two-channel micro pulse RBSA-lidar has been demonstrated continuous measurements of relative backscatter amplification in a turbulent atmosphere. Now it is sure that the diurnal variation of the received series of lidar data is consistent with the data from the meteorological observations and does not contradict to the atmospheric surface layer physics. For the stable temperature stratification at night time and for unstable stratification in the day time the relative backscatter amplification can have a fairly large and approximately the same values. It can be argued that the RBSA coefficient depends on the state of the atmosphere and reflects somehow this state. At the same time, this coefficient depends on the system parameters of the transceiver such as the beam size and the receiver size. We hope that the theory of RBSA-lidar will be developed.

\section{ACKNOWLEDGMENTS}

The authors many thank Dr. Sergei Odintsov and Dr. Vladimir Gladkikh for kindly providing meteo data of the ultrasonic anemometer.

This study was supported by the Russian Scientific Foundation for Maintenance and Development (Project: 14-17-00386).

\section{REFERENCES}

[1] Vinogradov A. G., Kravtsov Yu. A., and Tatarskii V. I., "The Effect of Intensification of Back Scattering by Bodies That Are Situated in a Medium Having Random Inhomogeneities," Radiophys. Quant. Electr. 16 (7), 818-823 (1973).

[2] Kravtsov Yu.A. and Saichev A.I., "Effects of Double Passage of Waves in Randomly Inhomogeneous Media," Physics-Uspekhi 25 (7), 494-508 (1982).

[3] Banakh V. A. and Mironov V. L., Lidar in a Turbulent Atmosphere (Artech House, Boston and London, 1987).

[4] Razenkov, I. A., Banakh V. A., Nadeev A.I. Aerosol Lidar for the Relative Backscatter Amplification Measurements, 27th International Laser Radar Conference, July 5-10, 2015, present proceedings, New York, USA.

[5] Gladkikh V.A, Makienko A.E. Digital ultrasonic meteostation. // Devices. 2009. V.109. No. 7. P. 21-25 (in Russian).

[6] Gladkikh V.A, Mamyshev V.P., Odintsov S.L. Experimental evaluation of structural constant of refractive index in the atmospheric surface layer. // Atmospheric and oceanic optics. 2015. V. 28. No. 4. P. 309-318 (in Russian).

[7] Laser Beam Propagation in the Atmosphere. Edited by J. W. Strohbehn. Springer Series Topics in Applied Physics, Vol. 25. (Berlin, Heidelberg, New York: Springer-Verlag, 1978). 